\title{
Erratum to: Contribution of indole-3-acetic acid in the plant growth promotion by the rhizospheric strain Bacillus amyloliquefaciens SQR9
}

\author{
Jiahui Shao • Zhihui Xu • Nan Zhang • Qirong Shen • \\ Ruifu Zhang
}

Published online: 8 January 2015

(C) Springer-Verlag Berlin Heidelberg 2015

\section{Erratum to: Biol Fertil Soils}

DOI 10.1007/s00374-014-0978-8

The original version of this article unfortunately contained the incorrect corresponding address of the corresponding author. The correct corresponding address should be:

College of Resources and Environmental Science, Nanjing Agricultural University, Nanjing, 210095, China. E-mail: rfzhang@njau.edu.cn Tel:86-025-84396477, Fax: 86-25843962

The online version of the original article can be found at http://dx.doi.org/ 10.1007/s00374-014-0978-8.

J. Shao $\cdot$ Z. Xu $\cdot$ N. Zhang $\cdot$ Q. Shen $\cdot$ R. Zhang

National Engineering Research Center for Organic-based Fertilizers, and Jiangsu Collaborative Innovation Center for Solid OrganicWaste Resource Utilization, Nanjing Agricultural University,

Nanjing 210095, People's Republic of China

\section{R. Zhang}

Key Laboratory of Microbial Resources Collection and Preservation, Ministry of Agriculture, Institute of Agricultural Resources and Regional Planning, Chinese Academy of Agricultural Sciences, Beijing 100081, People's Republic of China

R. Zhang $(\bowtie)$

College of Resources and Environmental Science, Nanjing Agricultural University, Nanjing 210095, China

e-mail: rfzhang@njau.edu.cn 